\title{
Excitons in atomically thin black phosphorus
}

\author{
A. Surrente, ${ }^{1}$ A. A. Mitioglu,,${ }^{1,2,{ }^{*}}$ K. Galkowski, ${ }^{1}$ W. Tabis,${ }^{1,3}$ D. K. Maude, ${ }^{1}$ and P. Plochocka ${ }^{1, \dagger}$ \\ ${ }^{1}$ Laboratoire National des Champs Magnétiques Intenses, UPR 3228, CNRS-UGA-UPS-INSA, Grenoble and Toulouse, France \\ ${ }^{2}$ Institute of Applied Physics, Academiei Stradă 5, Chisinau, MD-2028, Republic of Moldova \\ ${ }^{3}$ AGH University of Science and Technology, Faculty of Physics and Applied Computer Science, Aleja Mickiewicza 30, \\ PL-30-059 Krakow, Poland
}

(Received 2 January 2016; revised manuscript received 11 February 2016; published 15 March 2016)

\begin{abstract}
Raman scattering and photoluminescence spectroscopy are used to investigate the optical properties of single layer black phosphorus obtained by mechanical exfoliation of bulk crystals under an argon atmosphere. The Raman spectroscopy, performed in situ on the same flake as the photoluminescence measurements, demonstrates the single layer character of the investigated samples. The emission spectra, dominated by excitonic effects, display the expected in-plane anisotropy. The emission energy depends on the type of substrate on which the flake is placed due to the different dielectric screening. Finally, the blueshift of the emission with increasing temperature is well described using a two-oscillator model for the temperature dependence of the band gap.
\end{abstract}

DOI: 10.1103/PhysRevB.93.121405

Black phosphorus, the most stable of all the allotropes of phosphorus, has been intensively studied by different experimental methods from the early 1950's [1-7]. Bulk black phosphorus is a semiconductor, with a band gap of about $0.335 \mathrm{eV}[1,4]$. The orthorhombic bulk crystal has a layered structure, with atomic layers bound by weak van der Waals interactions. A single atomic layer is puckered, with the phosphorus atoms being parallel in the $(010)$ plane $[3,8,9]$. Atomically thin monolayers have been recently isolated using mechanical exfoliation [10], adding black phosphorus to the rapidly growing family of emerging two-dimensional materials. The band gap of black phosphorus is always direct and can be tuned from $0.3 \mathrm{eV}$ to the visible part of the spectrum [11,12]. In contrast, graphene is gapless [13], and the transition metal dichalcogenides (TMDs) have an indirect gap in the bulk phase and only monolayer TMDs have a direct gap [14]. Moreover, black phosphorus exhibits a strong in-plane anisotropy [11,12,15-17], which is absent in graphene and TMDs. Additionally, the relatively high mobilities measured at room temperature, combined with the direct band gap, result in an on/off ratio for field effect transistors of the order of $10^{5}[12,18,19]$.

Although black phosphorus has a wide range of possible applications, including tunable photodetectors [20], field effect transistors [12,16,18,19], or photon polarizers [11,12,15-17], many of its electronic properties are not yet fully understood. The main difficulty arises from the sensitivity of black phosphorus to its environment, notably its high reactivity when exposed to air and laser light [10,21,22]. Theoretical calculations predict a band gap of monolayer black phosphorus ranging from $1 \mathrm{eV}[11,19]$ to $2.15 \mathrm{eV}[10,11]$, with a binding energy of the neutral exciton of $0.8 \mathrm{eV}$ in vacuum [11] and of $0.38 \mathrm{eV}$ when placed on $\mathrm{SiO}_{2}$ substrate [10]. The measured photoluminescence (PL) emission energy from bilayer black phosphorus is between $1.2 \mathrm{eV}$ [23] and $1.6 \mathrm{eV}$ [10], while

\footnotetext{
*Present address: High Field Magnet Laboratory (HFML-EMFL), Institute for Molecules and Materials, Radboud University, Toernooiveld 7, 6525 ED Nijmegen, The Netherlands.

†paulina.plochocka@lncmi.cnrs.fr
}

monolayer black phosphorus shows neutral exciton emission around $1.3 \mathrm{eV}$ [24], $1.45 \mathrm{eV}$ [19], or $1.76 \mathrm{eV}$ [25]. The latter value was related to the simultaneous observation of charged exciton emission at around $1.62 \mathrm{eV}$ [25]. Using scanning tunneling microscopy, the band gap of a single layer of black phosphorus was estimated to be $2.05 \mathrm{eV}$ [26]. The exciton binding energy and consequently the emission energy strongly depend on the dielectric environment (substrate) [10,27]. This could partially explain the wide range of values for the black phosphorus emission energy found in the literature, possibly related also to a slightly different composition of the $\mathrm{SiO}_{2}$ substrates employed. Moreover, owing to the limited lifetime of the samples, the various characterization techniques used to identify monolayer black phosphorus (e.g., atomic force microscopy, Raman spectroscopy) could not always be performed on the same flake where the optical response was investigated.

In this Rapid Communication we present a systematic investigation of the optical properties of monolayers of black phosphorus. We analyze the properties of the emission as a function of the dielectric constant of the substrate, excitation power, polarization, and temperature. The single layer character of the investigated flakes is demonstrated using in situ Raman measurements on the same flake used for the PL. We show that the PL emission energy of monolayer black phosphorus depends on the substrate used. The PL spectra, dominated by excitonic effects, exhibit the expected in-plane anisotropy. Finally, the blueshift of the emission energy with an increasing temperature is well described with a two-oscillator model for the temperature dependence of the band gap.

Single and few layer black phosphorus flakes have been obtained by mechanical exfoliation of a bulk crystal, purchased from Smart Elements (99.998\% nominal purity). The mechanical exfoliation was performed in a glove box filled with argon (Ar) gas $\left(<1 \mathrm{ppm} \mathrm{O}_{2},<1 \mathrm{ppm} \mathrm{H}_{2} \mathrm{O}\right)$. The flakes were subsequently transferred onto a $\mathrm{Si}$ substrate, in most cases capped with a $300 \mathrm{~nm}$ thick layer of $\mathrm{SiO}_{2}\left(\mathrm{Si} / \mathrm{SiO}_{2}\right.$ hereafter $)$. The samples were stored in vials, in the glove box, before their transfer to the cryostat under an Ar atmosphere.

For the optical measurements, the samples were mounted on the cold finger of a He-flow cryostat, which was then rapidly 
pumped to a pressure below $1 \times 10^{-4}$ mbar, minimizing the exposure of the black phosphorus to air. The excitation was provided by a frequency-doubled diode laser, emitting at $532 \mathrm{~nm}$ and focused on the sample by a $50 \times$ microscope objective ( 0.55 numerical aperture), yielding a spot size of $\sim 1 \mu \mathrm{m}$. The PL and Raman signals were collected through the same objective and analyzed by a spectrometer equipped with a liquid nitrogen cooled Si CCD camera.

Raman spectroscopy, which has been shown to be a very precise tool for the determination of the number of layers in TMDs [14,28-31] and in graphene [32,33], can also be used to identify monolayer black phosphorus. Bulk black phosphorus belongs to the $D_{2 h}^{18}$ space group. Of the 12 normal modes at the $\Gamma$ point of the Brillouin zone, six are Raman active [7,34]. The two $B_{3 g}$ modes are forbidden in the backscattering configuration and the $B_{1 \mathrm{~g}}$ mode at $194 \mathrm{~cm}^{-1}$ is very weak. Therefore, only three Raman modes are expected for bulk black phosphorus: $A_{g}^{1}, A_{g}^{2}$, and $B_{2 g}$ at around 365,470 , and $442 \mathrm{~cm}^{-1}$, respectively [7,34]. The $A_{g}^{2}$ and $B_{2 g}$ modes are related to the in-plane vibrations of the atoms, while the $A_{g}^{1}$ mode is related to the out-of-plane movement of the atoms [7,34].

Typical calibrated micro-Raman ( $\mu$ Raman) spectra of the bulk and single layer black phosphorus are presented in Fig. 1. For the bulk crystal three strong peaks are observed at 363.7, 440.3 , and $467.5 \mathrm{~cm}^{-1}$, corresponding well to the expected main Raman modes. A further peak was systematically observed at $520 \mathrm{~cm}^{-1}$ (Raman mode of the Si substrate), confirming the correct calibration of our Raman setup. The slight shift towards lower frequencies, compared to the previously cited literature values, can be related to the low temperature (4 K) at which our measurements have been carried out [23]. Under low excitation power $(17 \mu \mathrm{W})$, initially used to avoid any risk of inducing damage by exposure to the laser light, the Raman spectrum of the monolayer flake is similar to that of the bulk crystal. This suggests that the monolayer black phosphorus remains crystalline and no oxidization occurred during the transfer of the sample from the glove box to the cryostat. Compared to bulk, the $A_{g}^{1}$ mode does not shift within

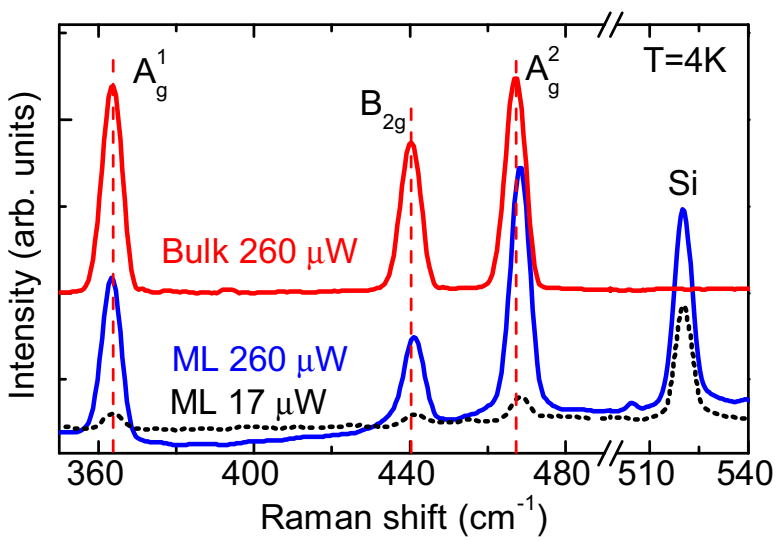

FIG. 1. Typical $\mu$ Raman spectra of bulk and single layer black phosphorus on $\mathrm{Si} / \mathrm{SiO}_{2}$ measured at $T=4 \mathrm{~K}$ for different excitation powers, as indicated. The dashed lines indicate the position of the three Raman modes $A_{g}^{1}, A_{g}^{2}$, and $B_{2 g}$ in bulk black phosphorus. experimental error. Although this Raman mode was found to soften in a monolayer sample [19], it was generally found to be rather insensitive to the number of layers $[35,36]$. In contrast, the position of $A_{g}^{2}$ and $B_{2 g}$ Raman modes shift to higher frequencies in the monolayer flake. The $A_{g}^{2}$ mode shifts by $1.7 \pm 0.2 \mathrm{~cm}^{-1}$ while the $B_{2 g}$ shifts by $1.0 \pm 0.2 \mathrm{~cm}^{-1}$. The change of the energy of the Raman modes as a function of the number of layers in black phosphorus has been already shown for both high $[10,19,35]$ and low frequency modes [37]. The shifts we measure are in good agreement with the reported values for single layer black phosphorus $\left(\sim 1.9 \mathrm{~cm}^{-1}\right.$ and around $\sim 1.0 \mathrm{~cm}^{-1}$ for $A_{g}^{2}[10,19,35,36]$ and $B_{2 g}[10,35]$, respectively), demonstrating the single layer character of the investigated flake. Raman measured at high excitation power (260 $\mu \mathrm{W}$, the same as used for the bulk crystal) does not show any additional features; the peak positions remain the same as for the data obtained for low excitation power. Thus there is no sign of laser-induced chemical modification of our samples [10], which is important since the excitation intensity used here is comparable to that used in our micro-PL ( $\mu \mathrm{PL})$ spectroscopy measurements.

In Fig. 2(a) we show representative low temperature $\mu \mathrm{PL}$ spectra for the monolayer flake previously characterized using Raman spectroscopy. The measurement was performed without removing the flake from the cryostat, which was maintained under vacuum. The spectrum is dominated by a very strong emission line centered at around $2 \mathrm{eV}$, identified with neutral exciton recombination. This line is consistently observed at this energy for all flakes transferred to $\mathrm{Si} / \mathrm{SiO}_{2}$ substrates. The typical full width at half maximum is of $\lesssim 90 \mathrm{meV}$ (line broadening induced by scattering with vacancies and impurities in the exfoliated flakes). The observed value of the exciton recombination energy is relatively high as compared to the theoretical band gap of $2.15 \mathrm{eV}$ computed for a black phosphorus single layer $[10,11]$, suggesting that the investigated sample is indeed a single layer. This is somewhat larger than the values already reported for black phosphorus single layers of $\simeq 1.3-1.76 \mathrm{eV}[19,24,25]$. This could be partly
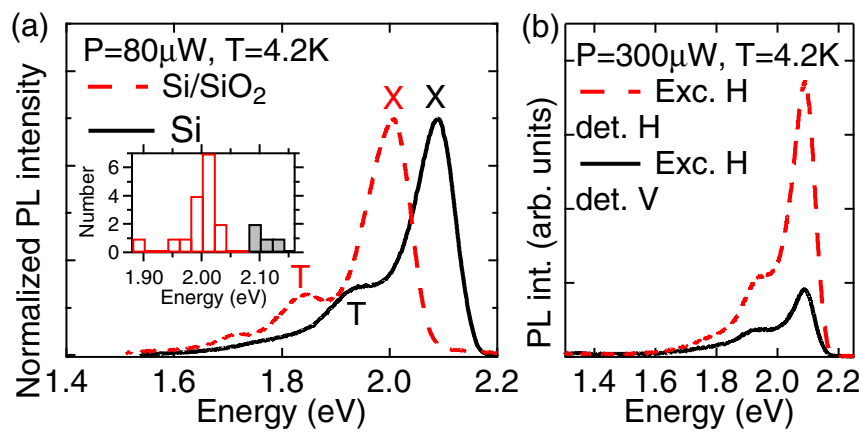

FIG. 2. (a) Low temperature $\mu \mathrm{PL}$ spectra of black phosphorus flakes on different substrates. $\mathrm{X}$ labels the neutral exciton transition, $\mathrm{T}$ the charged exciton. The inset shows a histogram of the emission energy for the 21 flakes investigated. The mean emission energy is $1.991 \mathrm{eV}$ with a standard deviation $\sigma=0.036 \mathrm{eV}$ for flakes on a $\mathrm{Si} / \mathrm{SiO}_{2}$ substrate and $2.102 \mathrm{eV}$ with $\sigma=0.015 \mathrm{eV}$ for flakes on a Si substrate. (b) Polarization-resolved $\mu \mathrm{PL}$ spectra of a black phosphorus flake on Si. 
ascribed to the different stoichiometry of the $\mathrm{SiO}_{2}$ layers used as a substrate, which induces a shift in the emission because of the different dielectric constant of the surrounding medium [38]. To verify this hypothesis, we have transferred black phosphorus flakes onto a $\mathrm{Si}$ substrate covered by a thin $(\sim 2 \mathrm{~nm})$ layer of native oxide. Such a substrate has a larger dielectric constant than the standard $\mathrm{Si} / \mathrm{SiO}_{2}$ substrates. Thus, the exciton binding energy is expected to be lower and the emission energy should be blueshifted. A typical $\mu \mathrm{PL}$ spectrum of a flake transferred onto a $\mathrm{Si}$ substrate is shown in Fig. 2(a). While the main spectral features resemble those of a flake on a $\mathrm{Si} / \mathrm{SiO}_{2}$ substrate, there is a large shift of the emission energy of $\sim 80 \mathrm{meV}$. This confirms the dependence of the emission energy on the dielectric environment, in agreement with previous results [10,27]. The effect of the dielectric environment seems to be stronger than for atomically thin TMDs, in agreement with theory and experiment [39]. However, we note that the effect of the dielectric environment alone is too small to account for the large variation of the emission energy reported in the literature $[19,24,25]$.

In the spectrum measured on a $\mathrm{Si} / \mathrm{SiO}_{2}$ substrate, in addition to the neutral exciton recombination, we observed additional features at $\sim 1.84 \mathrm{eV}$, which can be attributed to charged exciton recombination (systematically present in all the investigated flakes) [25] together with a low energy peak, typically at $\sim 1.72 \mathrm{eV}$, and possibly related to excitons bound to impurities. The low energy feature is not always present [see, e.g., the $\mu$ PL spectrum on a Si substrate in Fig. 2(a)].

Because of the low symmetry of the crystal structure and the screening in black phosphorus $[11,40]$, the exciton wave function is expected to be squeezed along the armchair direction, resulting in a polarization-dependent PL emission [24]. The linear polarization dependence of the emission of our flakes was investigated by mounting polarizers/analyzers in both the excitation and the detection paths. For any fixed orientation of the analyzer in the detection path, the detected signal is always stronger for horizontally $(\mathrm{H})$ polarized excitation. This stems from the polarization-dependent absorption properties of black phosphorus [16]. Regardless of the polarization of the excitation beam, whenever the analyzer in the detection path was set to $\mathrm{H}$, the intensity of the detected signal was a maximum, which is consistent with the strongly anisotropic nature of the exciton in black phosphorus [24]. For example, in Fig. 2(b), we show $\mu$ PL spectra excited with a H-polarized laser light and detected with either $\mathrm{H}$ or vertical (V) direction of the analyzer.

The evolution of the $\mu \mathrm{PL}$ spectra as a function of the excitation power gives information concerning the nature of the observed transition lines. We have measured the power dependence of the $\mu \mathrm{PL}$ spectra for several black phosphorus monolayer flakes. In Fig. 3(a) we show representative spectra measured at low, intermediate, and high excitation powers $P$ at $T=4.2 \mathrm{~K}$. Even after exciting with $P>1 \mathrm{~mW}$, the emission efficiency did not decrease for any of the investigated samples, suggesting that our preparation method helps improve the stability of the exfoliated black phosphorus flakes. Two peaks appear in the spectra of Fig. 3(a) for all excitation powers used. The neutral exciton peak slightly blueshifts $(\lesssim 2 \mathrm{meV})$ at high excitation powers, possibly due to a localized heating effect induced by the high power of the incoming laser beam (see
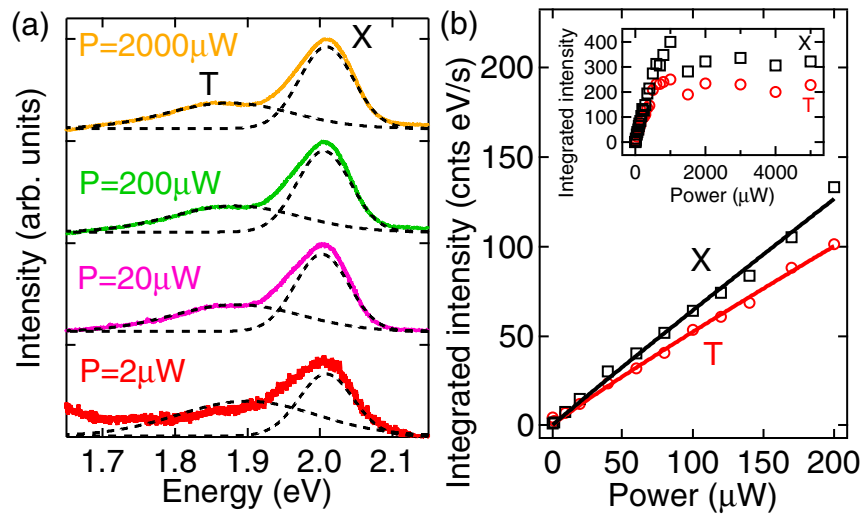

FIG. 3. (a) $\mu \mathrm{PL}$ spectra of a black phosphorus single layer on $\mathrm{Si} / \mathrm{SiO}_{2}$ measured at different excitation powers. The dashed curves are Gaussian fits used to calculate the integrated intensity. (b) Integrated intensity of the charged $(\mathrm{T})$ and neutral $(\mathrm{X})$ exciton emission versus excitation power. The solid lines are fits to a power law described in the text.

also below for a discussion of the temperature dependence of the black phosphorus PL).

In Fig. 3(b) we present the dependence of the integrated PL intensity $I$ of neutral and charged excitons as a function of the excitation power. At low excitation power, both intensities increase linearly with the power (indicating the absence of biexciton emission), as demonstrated by the fits to the power law $I \propto P^{n}$, with $n=0.97 \pm 0.03$ for the neutral exciton and $n=0.93 \pm 0.03$ for the charged exciton. At higher excitation power, both excitonic lines saturate at approximately the same level of excitation power $[P=1 \mathrm{~mW}$-see the inset to Fig. 3(b)], confirming that the two observed transitions are related to the recombination of a single electron-hole pair.

In view of the potential use of black phosphorus in a wide variety of electronic and optoelectronic applications, we have investigated the temperature dependence of its optical properties. In Fig. 4(a) we show normalized $\mu$ PL spectra measured at different temperatures. With increasing temperature, the charged exciton peak broadens. The neutral exciton emission blueshifts (measured $d E_{\mathrm{g}} / d T \sim 3.1 \times 10^{-4} \mathrm{eV} / \mathrm{K}$ between 40 and $160 \mathrm{~K}$ ), which is consistent with earlier reports of the temperature dependence of the band gap of bulk black phosphorus $\left(d E_{\mathrm{g}} / d T=2.8 \times 10^{-4} \mathrm{eV} / \mathrm{K}\right.$ [2] or $\left.2.33 \times 10^{-4} \mathrm{eV} / \mathrm{K}[41]\right)$. The behavior of the emission energy of the excitonic peak is shown in more detail in Fig. 4(b). In bulk semiconductors, the variation of the band gap as a function of the temperature is a direct consequence of the renormalization of the band gap via the electron-phonon interaction and of the thermal expansion of the lattice [42]. In the framework of the two-oscillator model [43-46], the band gap $E_{\mathrm{g}}$ is approximated by

$$
E_{\mathrm{g}}(T)=E_{0}+E_{1}\left(\frac{2}{e^{\frac{\hbar \omega_{1}}{k_{\mathrm{B}} T}}-1}+1\right)+E_{2}\left(\frac{2}{e^{\frac{\hbar \omega_{2}}{\mathrm{k}_{\mathrm{B}} T}}-1}+1\right),
$$

where $E_{0}$ is the bare band gap (i.e., the low temperature band gap exhibited in the absence of zero point motion), $E_{1}+E_{2}$ is the renormalization energy, and $\hbar \omega_{1}=17.23 \mathrm{meV}$ and $\hbar \omega_{2}=$ $52.82 \mathrm{meV}$ denote the two oscillator energies, as extracted 

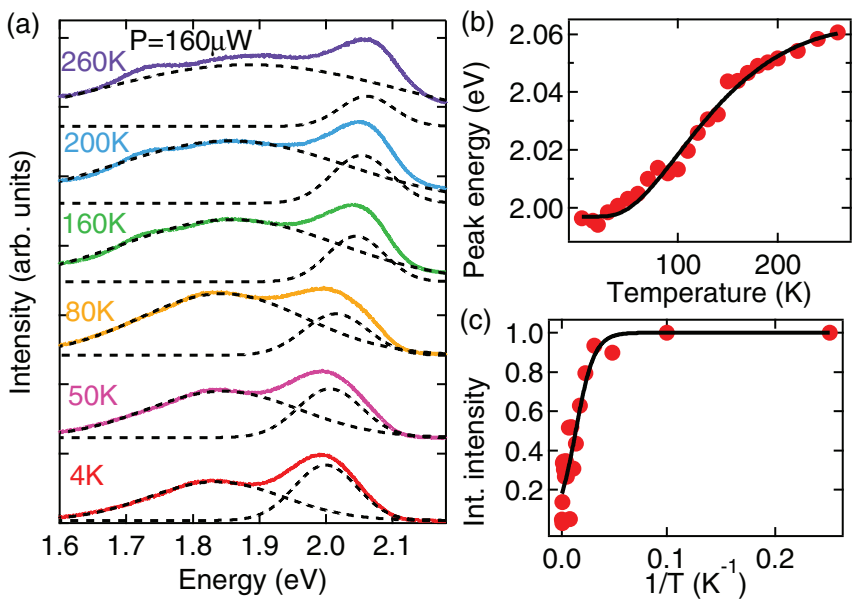

FIG. 4. (a) $\mu \mathrm{PL}$ spectra of a black phosphorus single layer on $\mathrm{Si} / \mathrm{SiO}_{2}$ measured at different temperatures. The dashed lines are Gaussian fits used to extract the emission energy. (b) Emission energy of the neutral exciton as a function of the temperature. (c) Integrated intensity of the neutral exciton versus temperature. The solid lines are fits to the models described in the text.

from the computed phonon density of states of monolayer black phosphorus [47]. The neutral exciton emission energy is then given by $E_{\mathrm{g}}(T)-E_{\mathrm{X}}$, where $E_{\mathrm{X}}$ is the exciton binding energy. The solid curve shown in Fig. 4(b) is obtained by fitting the experimental data, yielding $\left(E_{0}-E_{\mathrm{X}}\right)=2.22 \pm$ $0.02 \mathrm{eV}$ (larger than the observed low $T$ transition energy, suggesting the occurrence of band gap renormalization due to electron-phonon interaction), $E_{1}=72.6 \pm 0.3 \mathrm{meV}$, and $E_{2}=-297 \pm 3 \mathrm{meV}$.

The integrated intensity of the excitonic transition as a function of the inverse substrate temperature $T^{-1}$ is shown in Fig. 4(c). With increasing temperature, the emission intensity decreases, owing to the thermal activation of nonradiative recombination centers. To quantify the activation energy $E_{\mathrm{A}}$, the experimental data are fitted using $I(T) / I(T=0)=$
$1 /\left(1+a e^{-E_{\mathrm{A}} / \mathrm{k}_{\mathrm{B}} T}\right)$ [48], where $a$ is related to the ratio of the radiative and nonradiative lifetimes [49], to give $E_{\mathrm{A}}=$ $10 \pm 1 \mathrm{meV}$, and $a=6.8 \pm 2$. The significantly lower value of $E_{\mathrm{A}}$ as compared to the computed exciton binding energy [10] confirms that the decrease of the PL intensity at high $T$ is brought about by the thermal occupation of nonradiative recombination centers rather than the dissociation of the excitons.

In summary, we have performed a detailed investigation of the optical properties of monolayer black phosphorus mechanically exfoliated in an Ar atmosphere. No significant degradation of the PL emission was induced by the laser illumination, suggesting that this preparation method preserves the optical properties of the black phosphorus flakes. The shift of the Raman modes measured on the exfoliated flakes with respect to the bulk black phosphorus confirms the single layer character of the exfoliated black phosphorus flakes. The measured $\mu \mathrm{PL}$ spectra exhibit strong emission lines, attributed to the recombination of neutral and charged excitonic complexes. It is not clear if the enhanced emission energy, compared to the previously reported values [19,24,25], is linked with a larger intrinsic band gap or a reduced exciton binding energy. The excitonic nature of the observed PL was confirmed by the observed polarization dependence and by the nearly linear increase of the emission intensity with the excitation power. The increase in the emission energy with temperature was modeled with a two-oscillator model to account for the temperature dependence of the semiconductor band gap.

The authors gratefully acknowledge Baptiste Vignolle for his assistance with the glove box and for his careful proofreading and Geert Rikken for providing the bulk black phosphorus. A.A.M. acknowledges financial support from the French foreign ministry. This work was partially supported by ANR JCJC project milliPICS, the Région Midi-Pyrénées under Contract No. MESR 13053031, and STCU Project No. 5809.
[1] R. W. Keyes, Phys. Rev. 92, 580 (1953).

[2] D. Warschauer, J. Appl. Phys. 34, 1853 (1963).

[3] Y. Maruyama, S. Suzuki, K. Kobayashi, and S. Tanuma, Physica B+C 105, 99 (1981).

[4] H. Asahina, Y. Maruyama, and A. Morita, Physica B+C 117118, Part 1, 419 (1983).

[5] J. C. Jamieson, Science 139, 1291 (1963).

[6] J. Wittig and B. T. Matthias, Science 160, 994 (1968).

[7] S. Sugai and I. Shirotani, Solid State Commun. 53, 753 (1985).

[8] S. Narita, S. Terada, S. Mori, K. Muro, Y. Akahama, and S. Endo, J. Phys. Soc. Jpn. 52, 3544 (1983).

[9] Y. Takao, H. Asahina, and A. Morita, J. Phys. Soc. Jpn. 50, 3362 (1981).

[10] A. Castellanos-Gomez, L. Vicarelli, E. Prada, J. O. Island, K. L. Narasimha-Acharya, S. I. Blanter, D. J. Groenendijk, M. Buscema, G. A. Steele, J. V. Alvarez, H. W. Zandbergen, J. J. Palacios, and H. S. J. van der Zant, 2D Mater. 1, 025001 (2014).
[11] V. Tran, R. Soklaski, Y. Liang, and L. Yang, Phys. Rev. B 89, 235319 (2014).

[12] X. Ling, H. Wang, S. Huang, F. Xia, and M. S. Dresselhaus, Proc. Natl. Acad. Sci. USA 112, 4523 (2015).

[13] K. S. Novoselov, A. K. Geim, S. V. Morozow, D. Jiang, M. I. Katsnelson, I. V. Grigorieva, S. V. Dubonos, and A. A. Firsov, Nature (London) 438, 197 (2005).

[14] Q. H. Wang, K. Kalantar-Zadeh, A. Kis, J. N. Coleman, and M. S. Strano, Nat. Nanotechnol. 7, 699 (2012).

[15] T. Low, A. S. Rodin, A. Carvalho, Y. Jiang, H. Wang, F. Xia, and A. H. Castro Neto, Phys. Rev. B 90, 075434 (2014).

[16] F. Xia, H. Wang, and Y. Jia, Nat. Commun. 5, 4458 (2014).

[17] J. Qiao, X. Kong, Z.-X. Hu, F. Yang, and W. Ji, Nat. Commun. 5, 4475 (2014).

[18] L. Li, Y. Yu, G. J. Ye, Q. Ge, X. Ou, H. Wu, D. Feng, X. H. Chen, and Y. Zhang, Nat. Nanotechnol. 9, 372 (2014).

[19] H. Liu, A. T. Neal, Z. Zhu, Z. Luo, X. Xu, D. Tománek, and P. D. Ye, ACS Nano 8, 4033 (2014). 
[20] M. Buscema, D. J. Groenendijk, S. I. Blanter, G. A. Steele, H. S. J. van der Zant, and A. Castellanos-Gomez, Nano Lett. 14, 3347 (2014).

[21] S. P. Koenig, R. A. Doganov, H. Schmidt, A. H. Castro Neto, and B. Özyilmaz, Appl. Phys. Lett. 104, 103106 (2014).

[22] Z. X. Gan, L. L. Sun, X. L. Wu, M. Meng, J. C. Shen, and P. K. Chu, Appl. Phys. Lett. 107, 021901 (2015).

[23] S. Zhang, J. Yang, R. Xu, F. Wang, W. Li, M. Ghufran, Y.-W. Zhang, Z. Yu, G. Zhang, Q. Qin, and Y. Lu, ACS Nano 8, 9590 (2014).

[24] X. Wang, A. M. Jones, K. L. Seyler, V. Tran, Y. Jia, H. Zhao, H. Wang, L. Yang, X. Xu, and F. Xia, Nat. Nanotechnol. 10, 517 (2015).

[25] J. Yang, R. Xu, J. Pei, Y. W. Myint, F. Wang, Z. Wang, S. Zhang, Z. Yu, and Y. Lu, Light: Sci. Appl. 4, e312 (2015).

[26] L. Liang, J. Wang, W. Lin, B. G. Sumpter, V. Meunier, and M. Pan, Nano Lett. 14, 6400 (2014).

[27] A. H. Woomer, T. W. Farnsworth, J. Hu, R. A. Wells, C. L. Donley, and S. C. Warren, ACS Nano 9, 8869 (2015).

[28] C. Lee, H. Yan, L. E. Brus, T. F. Heinz, J. Hone, and S. Ryu, ACS Nano 4, 2695 (2010).

[29] H. R. Gutiérrez, N. Perea-Lpez, A. L. Elías, A. Berkdemir, B. Wang, R. Lv, F. Lpez-Urías, V. H. Crespi, H. Terrones, and M. Terrones, Nano Lett. 13, 3447 (2013).

[30] H. Li, Q. Zhang, C. C. R. Yap, B. K. Tay, T. H. T. Edwin, A. Olivier, and D. Baillargeat, Adv. Funct. Mater. 22, 1385 (2012).

[31] S.-L. Li, H. Miyazaki, H. Song, H. Kuramochi, S. Nakaharai, and K. Tsukagoshi, ACS Nano 6, 7381 (2012).

[32] A. C. Ferrari, J. C. Meyer, V. Scardaci, C. Casiraghi, M. Lazzeri, F. Mauri, S. Piscanec, D. Jiang, K. S. Novoselov, S. Roth, and A. K. Geim, Phys. Rev. Lett. 97, 187401 (2006).
[33] A. Gupta, G. Chen, P. Joshi, S. Tadigadapa, and Eklund, Nano Lett. 6, 2667 (2006).

[34] S. Sugai, T. Ueda, and K. Murase, J. Phys. Soc. Jpn. 50, 3356 (1981).

[35] A. Favron, E. Gaufres, F. Fossard, A.-L. Phaneuf-L'Heureux, N. Y.-W. Tang, P. L. Levesque, A. Loiseau, R. Leonelli, S. Francoeur, and R. Martel, Nat. Mater. 14, 826 (2015).

[36] W. Lu, H. Nan, J. Hong, Y. Chen, C. Zhu, Z. Liang, X. Ma, Z. Ni, C. Jin, and Z. Zhang, Nano Res. 7, 853 (2014).

[37] X. Luo, X. Lu, G. K. W. Koon, A. H. C. Neto, B. Özyilmaz, Q. Xiong, and S. Y. Quek, Nano Lett. 15, 3931 (2015).

[38] J. H. Choi and M. S. Strano, Appl. Phys. Lett. 90, 223114 (2007).

[39] Y. Lin, X. Ling, L. Yu, S. Huang, A. L. Hsu, Y.-H. Lee, J. Kong, M. S. Dresselhaus, and T. Palacios, Nano Lett. 14, 5569 (2014).

[40] P. Li and I. Appelbaum, Phys. Rev. B 90, 115439 (2014).

[41] M. Baba, Y. Nakamura, K. Shibata, and A. Morita, Jpn. J. Appl. Phys. 30, L1178 (1991).

[42] M. Cardona and M. L. W. Thewalt, Rev. Mod. Phys. 77, 1173 (2005).

[43] L. Viña, S. Logothetidis, and M. Cardona, Phys. Rev. B 30, 1979 (1984).

[44] M. Cardona and R. K. Kremer, Thin Solid Films 571, 680 (2014).

[45] P. Dey, J. Paul, J. Bylsma, D. Karaiskaj, J. M. Luther, M. C. Beard, and A. H. Romero, Solid State Commun. 165, 49 (2013).

[46] H. J. Lian, A. Yang, M. L. W. Thewalt, R. Lauck, and M. Cardona, Phys. Rev. B 73, 233202 (2006).

[47] Y. Aierken, D. Çakır, C. Sevik, and F. M. Peeters, Phys. Rev. B 92, 081408 (2015).

[48] M. Leroux, N. Grandjean, B. Beaumont, G. Nataf, F. Semond, J. Massies, and P. Gibart, J. Appl. Phys. 86, 3721 (1999).

[49] Y. Fang, L. Wang, Q. Sun, T. Lu, Z. Deng, Z. Ma, Y. Jiang, H. Jia, W. Wang, J. Zhou, and H. Chen, Sci. Rep. 5, 12718 (2015). 